\title{
Traffic Aggregation for Malware Detection
}

Ting-Fang Yen

Michael K. Reiter

Carnegie Mellon University, University of North Carolina Pittsburgh, Pennsylvania

U.S.A.

at Chapel Hill,

Chapel Hill, North Carolina

U.S.A.

DIMVA 2008 


\section{Background}

- Stealthy malware: spyware, adware, bots, ....

- Subtle command/control system

- Organized malicious activities

- Spamming, hosting phishing sites, DDoS attacks

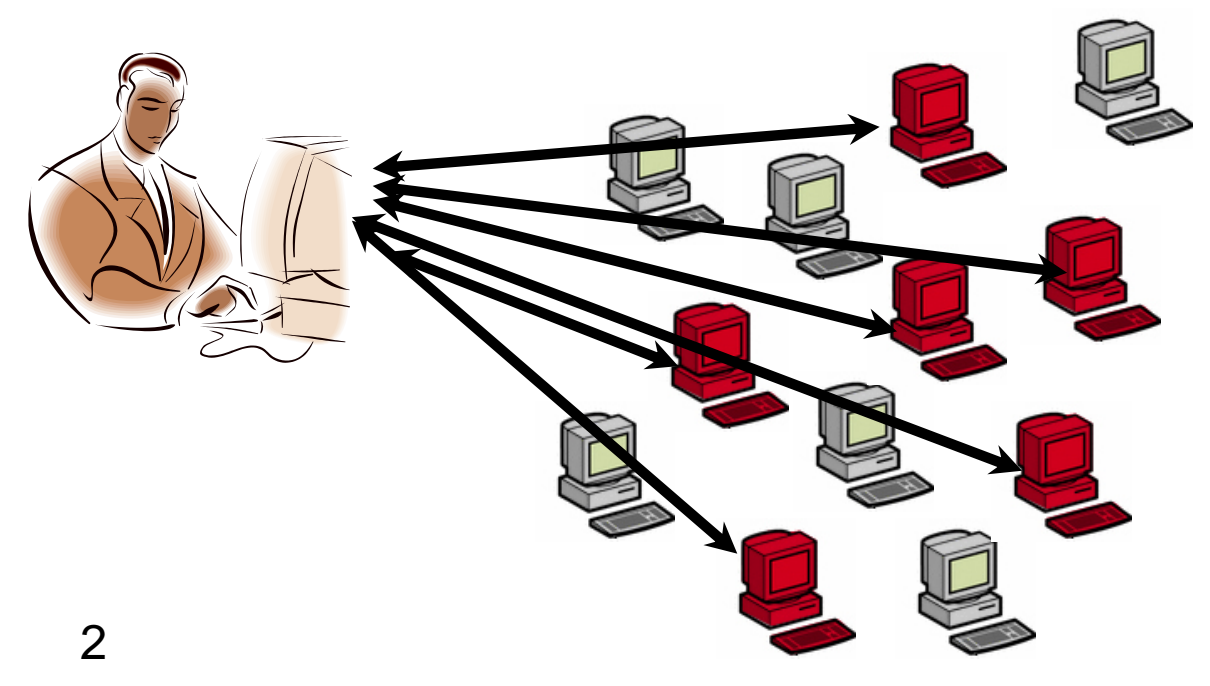




\section{Traffic Aggregation for}

Malware Detection (TAMD)

- Observe flow records at network border

- Assumptions:

- More than one infected host in the network

- Malware communication patterns different from benign hosts

- Traffic aggregates: network traffic sharing common characteristics

- Question: what characteristics can identify malware? 


\section{Aggregate Characteristics \\ - Common destination}

- Spyware "phone-home", botnet controller, bot update server, DDoS attack victim

- Similar Payload

- Bot commands

- Similar platform

- Platform-dependent infections

- Challenge: identify malware traffic while limiting the number ${ }_{4}$ of false alarms 


\section{Destination Aggregates}

- Internal hosts contacting the same "busierthan-usual" external subnets

- Use past traffic as baseline

- Represent internal hosts as vectors

- Dimensions (i.e., $D_{1}, D_{2}, \ldots$ ) correspond to external subnets

$\begin{array}{lllll}D_{1} & D_{2} & D_{3} & D_{4} & D_{5}\end{array}$

$$
\begin{aligned}
& \mathrm{H}_{1}=\left\langle\begin{array}{lllll}
1, & 1, & 0, & 1, & 1
\end{array}\right\rangle \\
& \mathrm{H}_{2}=\langle 1, \quad 1, \quad 1, \quad 0, \quad 0\rangle
\end{aligned}
$$

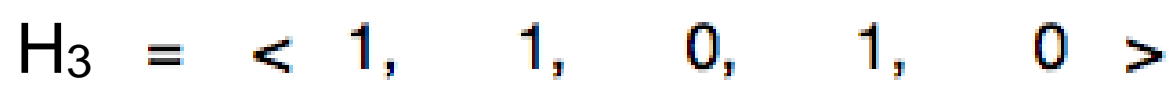




\section{Destination Aggregates}

\section{(cont'd)}

- Principal Component Analysis (PC

- Re-interpret data with new axes the captures most of the data variance

- Clustering

- Iteratively select furthest vector to be new hub

- Clusters contain hosts contacting the same "busier-than-usual" subnets 


\section{Destination Aggregates}

1

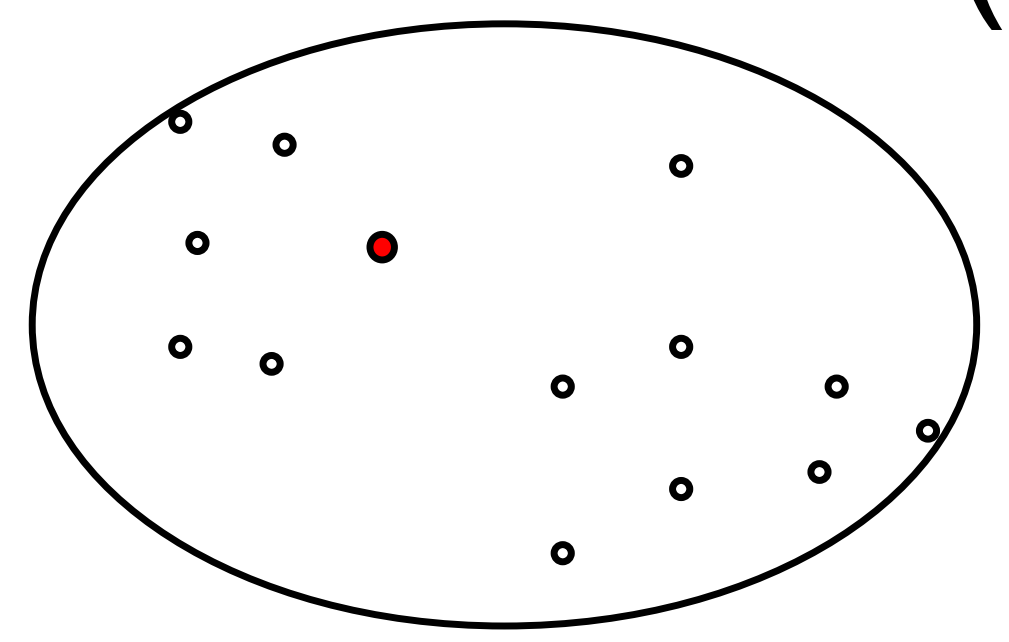

Assign random point as initial hub.

3

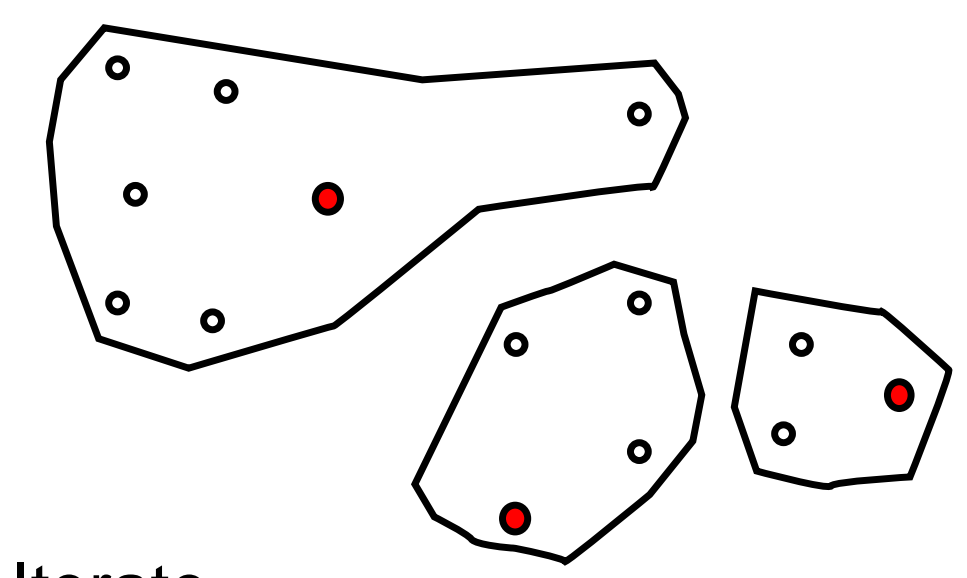

Iterate.

\section{(cont'd)}

2

Assign furthest point as new hub. Recluster.
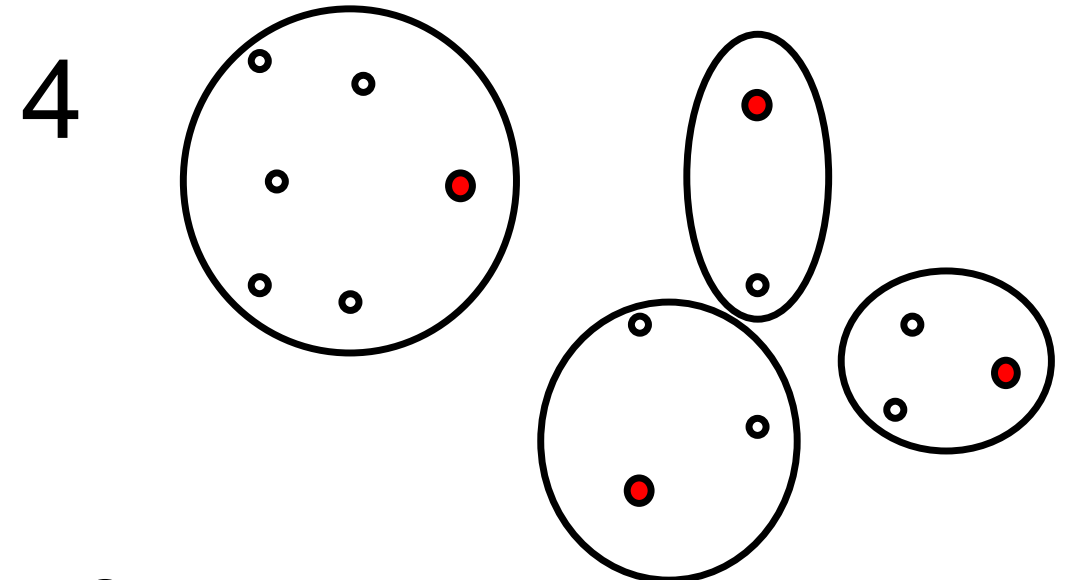

Stop when all points are closer to their hub than half of the average hub-hub distance. 


\section{Payload Aggregates}

- Flows with "similar" payload prefix

- Edit distance as similarity metric

- Number of character insertions, deletions, substitutions, to turn one string into the other

- Captures syntactic similarities

- ".bot.execute 1 notepad.exe"

".bot.execute $0 \mathrm{cmd}$.exe"

"abcdeeeeeenoopttuxx1... ."

- However, computationally expensive 


\section{Payload Aggregates (cont'd)}

- Locality Sensitive Hashing [Datar-Immorlica-Indyk-Mirrokni'04]

- Near-neighbor search: close points hash to same buckets

- Edit Sensitive Parsing [Cormode-Muthukrishnan'02 $\mathrm{P}$

- Embed edit distance into L1 distance ${ }^{\circ}{ }_{0}^{\circ}$

- As a result...

- Only compute edit distance for strings whose vectors hash to same buckets

- Time roughly proportional to size of data set 


\section{Platform Aggregates}

- Traffic from hosts of similar platform

- TTL (Time-to-Live) field

- Communication with characteristic sites

- e.g., Microsoft time server 


\section{Multi-Level Aggregation}

- Aggregation Functions:

- ByDestination

- ByPayload

- ByPlatform

- In combination, refine resulting aggregates

- Traffic sharing multiple relevant characteristics

- Example: platform-dependent infections that contact common sites 


\section{Aggregation Example}

Multiple infected hosts contacting sites uncommon to benign hosts.

\section{ByDestination}

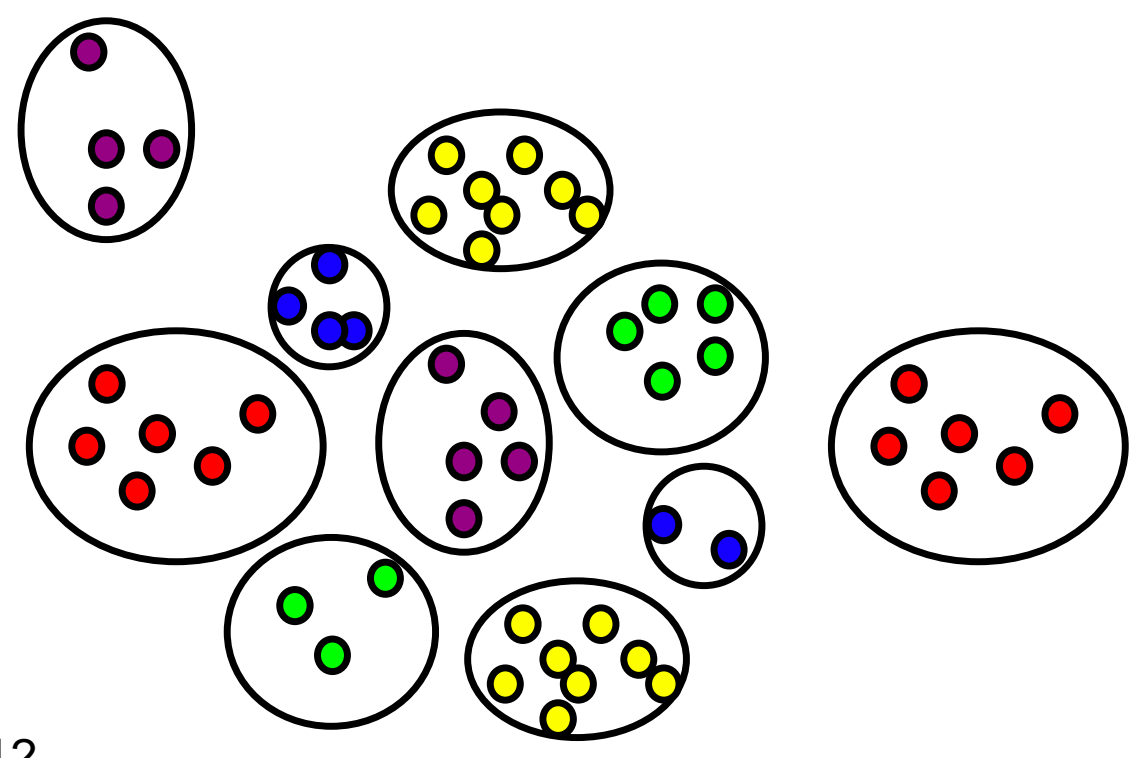


Aggregation Example (cont'd)

Multiple infected hosts contacting sites uncommon to benign hosts.

Malware communication similar among infected hosts.

\section{ByDestination}
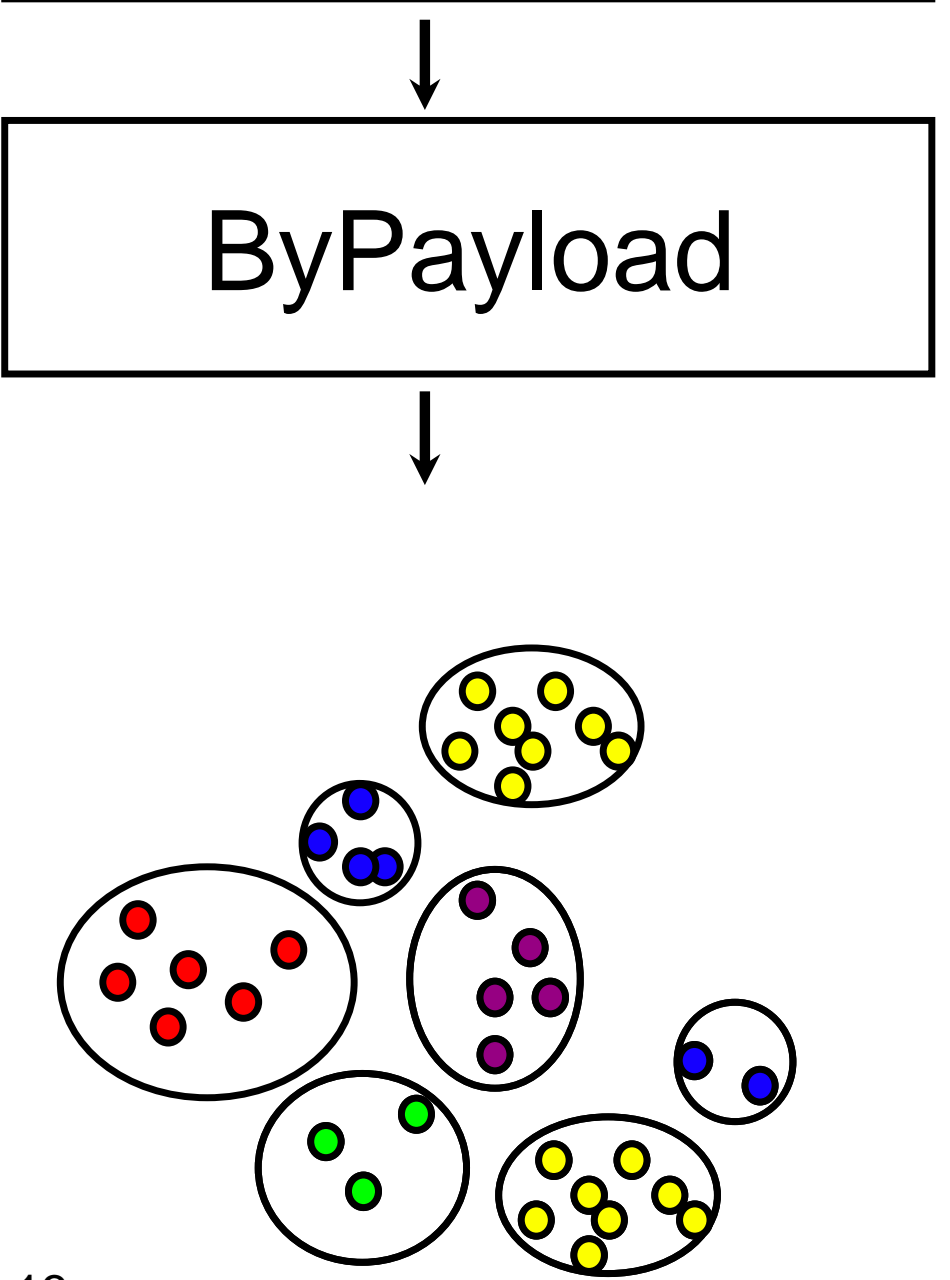


\section{Aggregation Example (cont'd)}

Multiple infected hosts contacting sites uncommon to benign hosts.

Malware communication similar among infected hosts.

Platform-dependent infection.






\section{Evaluation Data}

- Network traces from Carnegie Mellon University network border

- Two /16 subnets, over 33,000 hosts

\begin{tabular}{ll|l|l} 
- Argus flow records: & IP Header & Transport Header & Flow Attribute \\
\hline Source IP & Source Port & Byte Count \\
& Destination IP & Destination Port & Packet Count \\
& Protocol & TCP Sequence Number & Payload (64 bytes) \\
& TTL & TCP Window Size &
\end{tabular}

- Captures 5000 flows/sec

- 9 a.m. to 3 p.m. daily

- Experiments use TCP and UDP traffic only 


\section{Evaluation Data (cont'd)}

- Network traces from malware in virtual machines

- Bagle, IRCbot, Mybot, SDbot

- Infect 3 8 Windows XP virtual hosts with each malware binary

- One hour of traffic from each malware

- Network traces from botnets in honeynets

- Spybot : Four bots, 32-minute trace

- HTTP-bot : Four bots, three-hour trace

- Large botnet : > 340 bots, seven-minute trace 


\section{Evaluation}

- For every hour of campus traffic,

- For every malware,

- Assign malware traffic to randomly selected internal hosts of same platform

- Comprise $0.0097 \%$ of all internal hosts

- Input to aggregation functions

$\rightarrow \begin{gathered}\text { ByDestinatio } \\ \mathrm{n}\end{gathered} \rightarrow \begin{gathered}\text { ByPayloa } \\ \mathrm{d}\end{gathered} \rightarrow \begin{gathered}\text { ByPlatfor } \\ \mathrm{m}\end{gathered}$

- Repeat over every hour during three weeks in November/December 2007 


\section{Results}

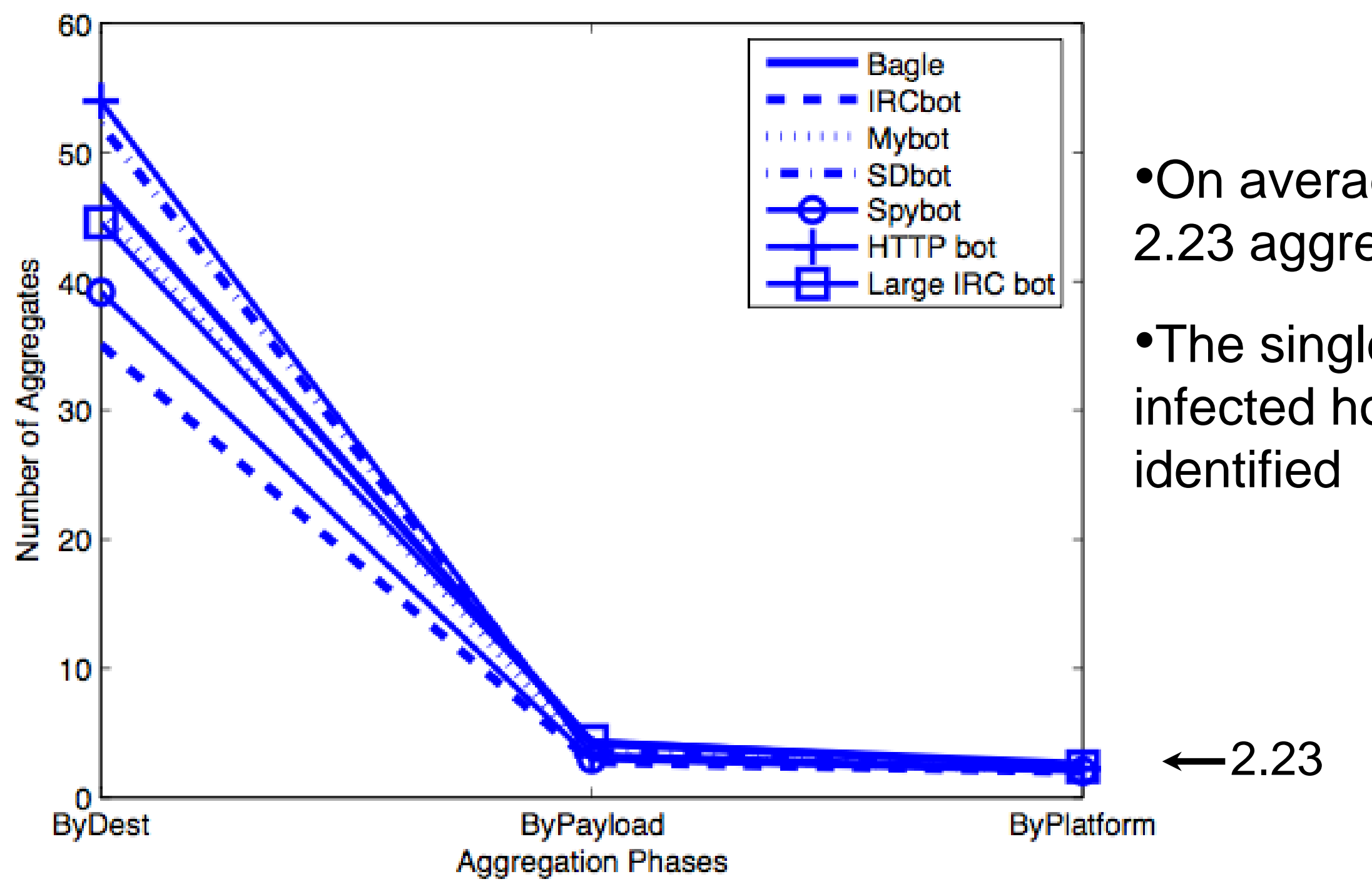




\section{Performance Statistics}

\section{Function Run Time}

\section{$120 \mathrm{sec}$ \\ Osec}

[ByDestination]

- Bagle

$\triangle$ Sdbot

- Large IRC bot
Total Run Time

$200 s$

$160 \mathrm{~s}$

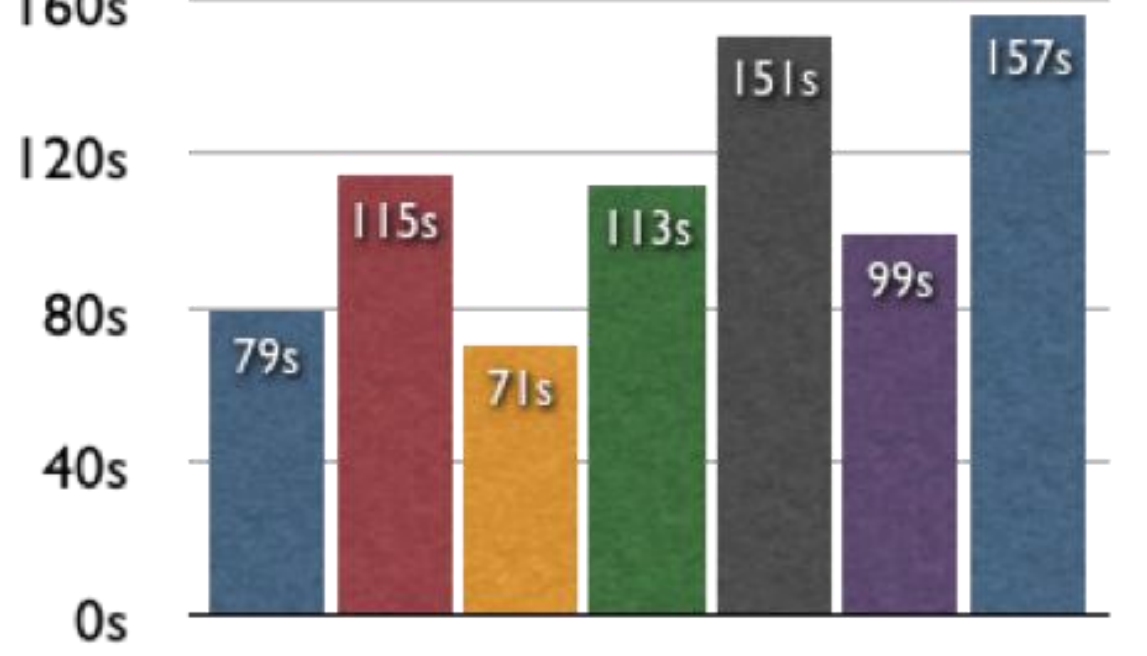

[ByPayload]

[ByPlatform]

ㅁ. IRCbot $\nabla$ Mybot

- Spybot O HTTPbot

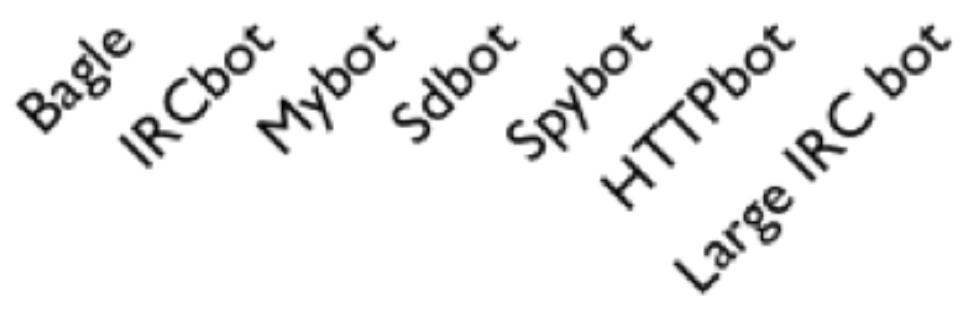




\section{Alternative Botnet Architectures}

- Peer-to-peer (P2P):

- Hard-coded peer list

- Bots report back to designated site

- Use P2P to transfer URLs for downloading binaries

- Hybrid: Smaller centralized botnets peer in P2P 


\section{Limitations and Ongoing}

- Temporal locality Wharkare communication

- But sparse communication restricts botnet size and responsiveness

- Diversity in hosts' platforms

- Good results with only ByDestination and ByPayload

- P2P with peer discovery through random probing

- ByPayload or ByPlatform

- Encrypted payload

- Extend "similar" to include encrypted traffic

- Isolated bots 


\section{Conclusion}

- Traffic Aggregation for Malware Detection (TAMD): Identifies traffic sharing common network characteristics

- Common destination

- Similar payload

- Common platform

- Detects stealthy platform-dependent malware contacting common sites

- Successful even when number of simulated infected hosts comprise $0.0097 \%$ of internal hosts 